\title{
Monogeusia for fructose, glucose, sucrose, and maltose
}

\author{
P. A. S. BRESLIN \\ Monell Chemical Senses Center, Philadelphia, Pennsylvania \\ G. K. BEAUCHAMP \\ Monell Chemical Senses Center and University of Pennsylvania \\ Philadelphia, Pennsylvania \\ and \\ E. N. PUGH, JR. \\ University of Pennsylvania, Philadelphia, Pennsylvania
}

\begin{abstract}
We investigated the ability of subjects to discriminate sugars with a whole-mouth forced-choice paradigm, in which a standard solution was compared with a test solution of varied concentration. Discrimination probabilities were U-shaped functions of test concentration: for 6 subjects and pairwise combinations of fructose, glucose, and sucrose, discriminability always declined to chance over a narrow range of test concentrations. At concentrations $\leq 100 \mathrm{mM}$, maltose was indiscriminable from fructose but discriminable at higher concentrations for 4 subjects. By analogy with the monochromacy of night vision, whereby any two lights are indiscriminable when their relative intensities are suitably adjusted, we call the gustatory indiscriminability of these sugars monogeusia. The simplest account of monogeusia is that all information about the indiscriminable sugars is represented by a single neural signal that varies only in magnitude. The discriminability of maltose from the other sugars at higher concentrations is consistent with the hypothesis that maltose also activates a second gustatory code.
\end{abstract}

The number of neural signals that encode information about sweet-tasting compounds has been vigorously debated (Bartoshuk, 1987; Beidler \& Tonosaki, 1985; Jakinovich \& Sugarman, 1989). One hypothesis is that the coding dimensionality for sugars is large, that is, many distinct signals are involved at every level of coding. Accordingly, each physically distinct sweet-tasting compound binds to a distinct population of receptors and produces a unique pattern of activity at the receptor level and at all subsequent levels of coding (Erickson, 1982; Faurion, 1987; Lawless \& Stevens, 1983; Schiffman, Cahn, \& Lindley, 1981). An alternative hypothesis is that the coding dimensionality of sugars is small, with only one or a small number of signals encoding information about sweet-tasting compounds (Birch, 1987; Breslin, Kemp, \& Beauchamp, 1994; McBurney, 1972; Shallenberger, 1993; von Skramlik, 1926).

Matching experiments have played an important role in uncovering the coding dimensionality of light stimuli for particular observers. For observers with normal color vision under photopic conditions, a light of arbitrary spectral distribution can be made indiscriminable from a par-

This work was supported by NIH Grants F32 DC00100 and RO1 DC00882 and by the Morley R. Kare fellowship. We are grateful to Sarah E. Kemp for advice and participation on portions of this work and to Karen Opdyke, Amy Fisher, Jeffrey Leen, and Jacqueline Ruttenberg for their technical assistance. Correspondence should be addressed to P. A. S. Breslin, Monell Chemical Senses Center, 3500 Market Street, Philadelphia, PA 19104 (e-mail: breslin@cattell.psych.upenn.edu). ticular mixture of three suitably chosen "primary light stimuli" (Wyszecki \& Stiles, 1982). Thus, day vision is said to be trichromatic, and the coding dimensionality of photopic stimuli is three. If attention is confined to visual stimuli comprising only wavelengths greater than $540 \mathrm{~nm}$ (the "Raleigh" region of the visible spectrum), all stimuli can be rendered indiscriminable from a suitable mixture of only two primaries, and thus observers are functionally $d i-$ chromatic. Under scotopic viewing conditions, any two lights can be rendered indiscriminable from one another simply by an adjustment of their relative intensities. Thus, in night vision, observers are monochromatic, and the coding dimensionality of scotopic stimuli is one (Pugh, 1988). The essential methodological feature of the visual experiments just described is that observers fail to discriminate a set of test stimuli from suitable mixtures of a special (but not unique) standard set, the "stimulus primary" set. The data of such experiments give rise to sets of "matching" stimuli, that is, stimulus equivalence classes to which all relevant stimuli of the class map. For example, in normal color vision, all visual stimuli map to a three-dimensional set, the so-called metamer classes. The number of stimulus primaries required to make matches to all the members of the set under standard viewing conditions is the coding dimensionality of the set.

To investigate the coding dimensionality of a group of simple saccharides, we used a two-alternative, forced-choice "duo-trio" taste discrimination protocol, and developed a novel analysis for the data. In each trial of the duo-trio 
paradigm, a subject was required to select which of three successively presented stimuli was the odd one; two of the three stimuli were identical. Each experiment comprised a series of trials for which there was a fixed standard stimulus, $S$, and a second test stimulus, $T$, whose concentration varied over trials. Experiment 1 examined the discriminability of the monosaccharides glucose and fructose. Experiment 2 tested the discriminability of a monosaccharide, fructose, from a disaccharide, sucrose. Experiment 3 examined the discriminability of fructose from a second disaccharide, maltose. Experiments 4 and 5 employed alternative psychophysical methods to test the generality of the findings of Experiments 1-3. Some of the data from Experiments 1 and 2 have been described briefly elsewhere (Breslin et al., 1994).

\section{GENERAL METHOD}

\section{Subjects}

Nonsmoking subjects of both sexes, between the ages of 21 and 35 , were recruited from the Monell staff, from Drexel University, and from the University of Pennsylvania and paid to participate in the experiments. The subjects were asked to refrain from eating within $2 \mathrm{~h}$ prior to testing. Prior to enrollment in an experiment, the protocol was explained to prospective subjects, who were then tested on 10 trials in which the discriminanda were 200 - and $600-\mathrm{mM}$ glucose. Subjects who did not discriminate perfectly were excused, on the assumption that they had abnormal taste or were unable to follow instructions.

\section{Stimuli}

All sugars were reagent-grade compounds and were purchased from major suppliers, as noted below. The sugars were dissolved in Naya spring water (Canada Dry) and maintained at $20^{\circ} \mathrm{C}$. Naya bottled water served both as the vehicle for all stimuli and as the adapting and rinsing stimulus in all experiments.

Naya water was chosen for the experiments because it was ranked the most neutral tasting of several bottled waters tested (Kemp \& Beauchamp, 1994). The ranking was obtained from a panel of 15 subjects, who were asked to order bottled waters, distilled water, and deionized water for "neutral taste." Naya water was given the highest average rank.

All solutions were kept at $20^{\circ} \mathrm{C}$ for at least $24 \mathrm{~h}$ prior to their use in the experiment to allow for complete mutarotation of the tautomers. The concentrations of the standards were chosen so that the stimuli would be roughly an order of magnitude above detection threshold (Pfaffmann, Bartoshuk, \& McBurney, 1971).

\section{Procedure: Duo-Trio Taste Discrimination Protocol}

The subjects participated in one session per day and 4-5 sessions per week. The subjects rinsed the whole mouth thoroughly four times with Naya bottled water prior to testing. For each session, consisting of 10 discrimination trials, a single taste standard, $S$, and a single test stimulus, $T$, were used; that is, both the chemical identities and the concentrations of $S$ and $T$ were held constant in an individual session. In the session, 10 sets of three cups ( $1 \mathrm{oz}$, Baxter) containing $10 \mathrm{ml}$ of solution were placed before a subject. A duo-trio trial consisted of the whole-mouth tasting of a set of three cups: the three cups contained two different solutions in one of four randomly chosen triplets: $\langle S S T\rangle,\langle T S S\rangle,\langle S T T\rangle,\langle T T S\rangle$. The observer's task was to choose whether the first or third stimulus was the odd stimulus of the triplet. The solutions were tasted from left cup to right cup, with Naya rinsing between each sampling. After each cup had been tasted once, the subject was instructed to retaste all stimuli from left to right again. The subjects rinsed twice between tasting each of the three stimuli of a trial, and at least four times in the 2-min interval between trials.
Completed discrimination functions for individual subjects typically involved 200-500 trials, in which a particular standard, $S$, with both a single chemical identity and a fixed concentration and a set of test stimuli, $\left\{T_{i}, i=1, \ldots, M\right\}$, of distinct chemical identity but varying concentration were presented over a series of testing sessions. Because of the time-consuming nature of the experiments and limited number of trials possible on each day, the following strategy was adopted to facilitate the collection of complete discrimination profiles. If, in the initial session in which a subject tasted a particular stimulus pair $\left(S, T_{i}\right)$, discrimination was perfect, that pair was not repeated; if the discrimination was less than perfect, the trial was repeated in one or more additional sessions, typically for $40-60$ trials over 4-6 sessions.

To minimize the number of experimental sessions needed to complete a discrimination profile, the total number, $M$, of different test stimuli was varied, depending on the taste standards; over all experiments, $M$ ranged from 9 to 17 . Typically, 20-50 sessions overall were needed to complete a profile. Different test concentrations were presented in a random order over sessions, with the exception that all subjects were given an initial starting concentration from the upper or lower end of the anticipated concentration range; this initial session provided an anchor point for demonstrating the difference between the stimuli for the subjects.

Olfactory cues provide a possible source of information in wholemouth discrimination experiments. To examine the possibility that subjects used olfactory cues for stimuli whose discriminability was initially found to be above chance in Experiments 3 and 5, the subjects were retested while wearing soft rubber noseclips (Rubbermaid). Noseclips have been shown to eliminate orthonasal and retronasal olfactory cues (Hettinger, Myers, \& Frank, 1990).

\section{Statistical Analyses}

Point-by-point chi-square analyses. We summarized the data of each experiment by plotting as a function of the test concentration, $T$, the estimates of a set of discrimination probabilities, $\{p$ (discriminate $T_{i}$ from $S$ ), $\left.i=1, \ldots, M\right\}$. In most cases, these discrimination functions were found to be U-shaped functions of the test concentration (e.g., Figures 1 and 2), with the nadir of the function near chance. To test the hypothesis that $p$ (discriminate $T_{i}$ from $S$ ) $=0.5$ for each data point, we employed a chi-square test, assuming the data for any given pair $\left(S, T_{i}\right)$ of discriminanda were Bernoulli trials. Different symbols were used to denote different significance levels, as described in the figure legends.

Parametric analysis of discrimination function. To characterize quantitatively the discrimination functions and further test the hypothesis that discriminability declines to chance for some particular concentration of the test, we employed the following analysis, which was patterned after the analysis used by Calkins, Thornton, and Pugh (1992) to characterize wavelength-discrimination data. The data of each experiment were fitted with a two-limbed "inverted Gaussian" discrimination function described by

$$
\begin{aligned}
& p \text { (discriminate } T \text { from } S \text { ) } \\
& \qquad=1-\delta \exp \left[-\frac{1}{2}\left(\frac{\log T-\log \mu}{\log \sigma^{-}}\right)^{2}\right], T \leq \mu \\
& =1-\delta \exp \left[-\frac{1}{2}\left(\frac{\log T-\log \mu}{\log \sigma^{+}}\right)^{2}\right], T>\mu
\end{aligned}
$$

(e.g., Figures 1, 2, and 4). In Equation 1, $T$ is the test stimulus concentration, $\mu$ is the concentration at which discriminability of test and standard is a minimum, $\delta$ is the depth of loss of discriminability, and $\sigma^{ \pm}$characterize the steepnesses of the descending and ascending sides of the discrimination function. One particularly useful feature of the application of Equation 1 is that in cases in which discriminability declines to chance the parameter $\mu$ provides an estimate based on the entire data set of the concentration of the test that "matches" the standard. A second useful feature is that the steepness 
parameters, $\sigma^{ \pm}$, can be used to obtain Weber fractions from the discrimination profiles, as shown below. A third important feature is that the null hypothesis that discrimination declines to chance in an experiment is captured in the parametric hypothesis $\delta=.5$, which can be tested with a well-known statistic, as we now explain.

The fitting of Equation 1 to the data was performed with a maximum-likelihood analysis. Given the parametric formula for the discrimination function in Equation 1, the likelihood function for each experiment is defined by the formula

$$
L\left(\mu, \sigma^{+}, \sigma^{-}, \delta \mid \text { data }\right)=\prod_{i=1}^{M} p_{i}^{n c_{i}}\left(1-p_{i}\right)^{N_{i}-n c_{i}}
$$

In Equation 2, $p_{i}=p\left(\right.$ discriminate $T_{i}$ from $S$ ) and $\left\{T_{i}, i=1, \ldots, M\right\}$ is the set of test stimuli used in the experiment, $N_{i}$ is the total number of trials for the pair $\left(S, T_{i}\right)$ of discriminanda, and $n c_{i}$ is the number of correct discrimination decisions for the same pair. By maximizing the likelihood function over the parameter space, we obtained maximum-likelihood estimates (MLEs) of the parameters of Equation 1. The maximization was done with a routine built on the Nelder-Mead simplex search algorithm of Matlab. Specifically, the negative logarithm of the likelihood function was maximized twice: first, in the general parameter space $\Omega=\{-\infty<\log \mu<+\infty, 0<$ $\left.\log \sigma^{-}, 0<\log \sigma^{+}, 0<\delta<1.0\right\}$, and second, in the parameter subspace $\omega \subseteq \Omega$, in which $\delta$ is held fixed at .5.

The null hypothesis that discriminability declines to chance in an experiment is equivalent to the parametric hypothesis $\delta=.5$. A statistical test of this parametric hypothesis can be developed in terms of the likelihood ratio statistic, $\hat{\lambda}=[\max L(\omega)] /[\max L(\Omega)]$. According to Wilks's (1962) theorem, under the null hypothesis $-2 \ln \hat{\lambda}$ is asymptotically distributed as $\chi^{2}$ with $1 \mathrm{df}$.

A related, but somewhat more complicated, maximum-likelihood analysis was developed to characterize the results of Experiment 4, in which the subjects made forced-choice same/different judgments for pairs of stimuli. That analysis will be described in the presentation of Experiment 4.

\section{EXPERIMENTS 1, 2, AND 3}

\section{Experiment 1}

In Experiment 1, we investigated whether subjects could discriminate fructose and glucose. Two discrimination series were used: one in which the $S=100-\mathrm{mM}$ fructose and a second in which $S=200-\mathrm{mM}$ fructose.

\section{Method}

Subjects. Two females and 1 male from the subject pool described in General Method served in Experiment 1.

Stimuli. D-fructose and $\alpha$-D-glucose (Aldrich) were dissolved in Naya spring water and maintained at $20^{\circ} \mathrm{C}$.

Procedure. The procedure was the duo-trio method described in General Method.

Analysis. The analysis was that described in General Method.

\section{Results}

Figure 1 shows the data of the 3 subjects. In each case, the estimated discrimination function, $p$ (discriminate $T$ from $S$ ), is a $U$-shaped function of $T$, declining approximately to chance. Five of the six discrimination functions yielded at least one point (large $\bullet$ ) having $p \geq .1$, thus failing to reject the null hypothesis with $p<.1$. The remaining discrimination function (Figure 1C) had three points with $.05<p<.1$ (small $\bullet$ ).
Table 1 gives the MLEs of the parameters of Equation 1 and the likelihood ratio statistics obtained from applying the analysis to the data of Experiments 1 and 2. These statistics will be examined after presentation of the data of Experiment 2.

\section{Experiment 2}

In Experiment 2, glucose, which was the test stimulus in Experiment 1, was tested for discriminability against the disaccharide sucrose.

\section{Method}

Subjects. Two females and 1 male served in Experiment 2.

Stimuli. Sucrose $(\alpha$-D-glucopyranosyl- $\beta$-D-fructofuranoside; Fisher), a disaccharide containing a glucose and a fructose moiety, and $\alpha$-D-glucose (Aldrich) were dissolved in Naya spring water and maintained as described above. The discrimination standards were 100 - and $200-\mathrm{mM}$ sucrose.

Procedure. The procedure was that described in General Method. Analysis. The analysis was that described in General Method.

\section{Results}

Figure 2 shows the data for the 3 subjects of Experiment 2. In each case, as in Experiment 1, discriminability was a U-shaped function of $T$, with a minimum near chance. Five of the six U-shaped functions of Figure 2 yielded at least one point (large $\bullet$ ), with $p \geq .1$, thus failing to reject the hypothesis that discrimination declines to chance. The remaining function (Figure $2 \mathrm{C}$ ) had two points with $.05<$ $p<.1$ (small •).

\section{Summary Analysis of Experiments 1 and 2}

\section{Loss of Discriminability or Artifact?}

Before the results of additional experiments are presented, a fundamental question must be addressed: Could the decline in performance to chance levels in Experiments 1 and 2 be due to methodological and/or statistical artifacts or is it truly due to loss of discriminability? The most straightforward argument based upon the data and analysis presented so far against a statistical or methodological artifact is this: if the declines in performance were artifacts, one would expect to find (for any particular pair of discriminanda) minima spread randomly over the test concentration axis; what we found for each pair, however, was a single minimum in a small region of test concentration. Put more formally, since the data points that fail to reject the hypothesis $p$ (discriminate $T$ from $S)=.5$ according to the chi-square tests were always near the minima of the discrimination functions, and since also there were always points on both sides of the minima that very strongly rejected the hypothesis, the failures to reject cannot be ascribed to inadequately sensitive experimental methodology, nor can they be ascribed to an overall lack of statistical power. Another argument against a methodological artifact is the consistency across observers in the values of $\mu$ for particular standards. Thus, the most reasonable conclusion is that the failures to reject the hypothesis are, in fact, due to loss of discriminability. (Additional 
Standard: $100 \mathrm{mM}$ fructose

Standard: $200 \mathrm{mM}$ fructose
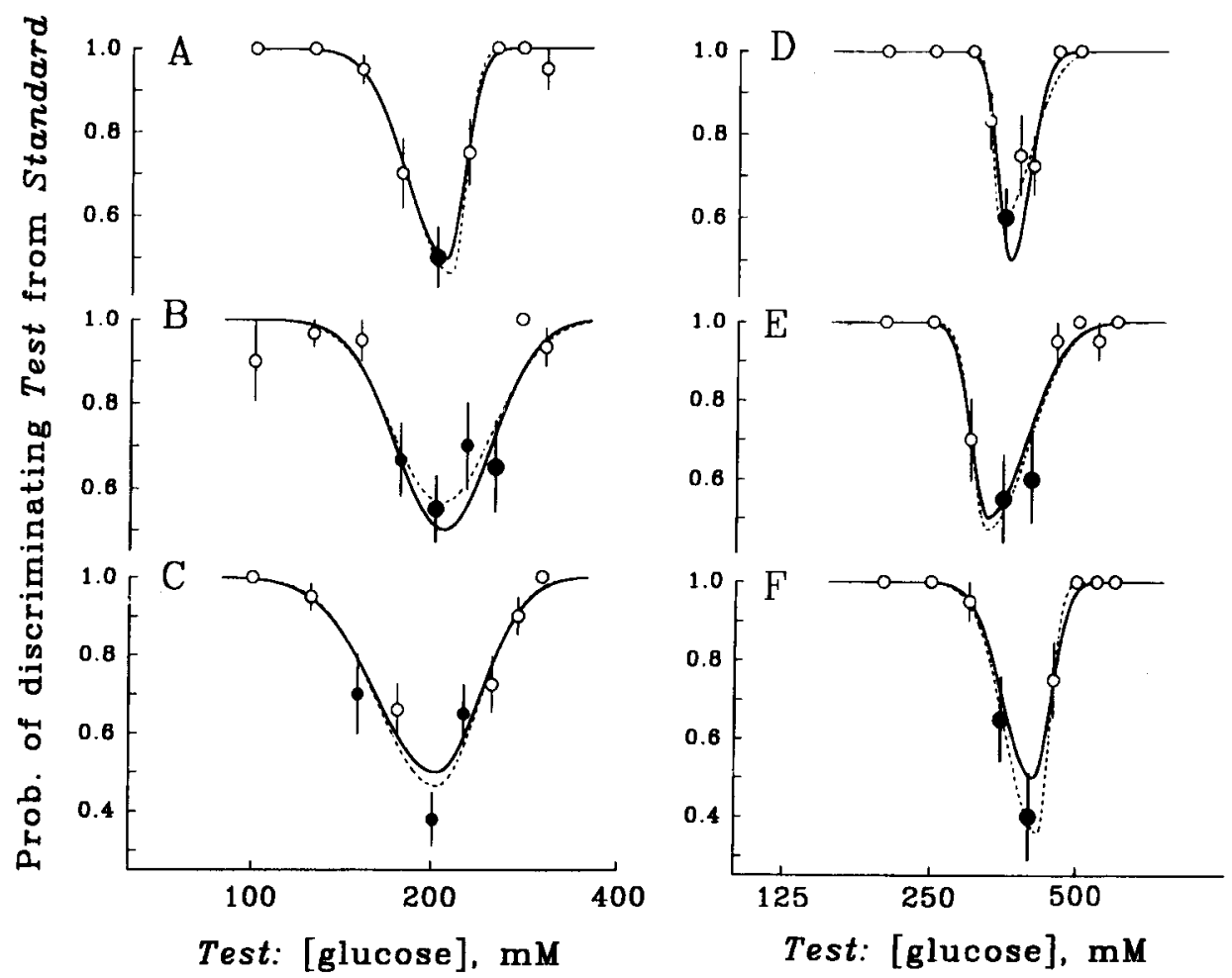

Figure 1. Discrimination functions of individual subjects for glucose test stimuli (abscissa) versus two fructose standards (labels above panels). Each row of two panels (e.g., A and D) presents the data of an individual subject. Symbols give proportions of correct responses in duo-trio trials; error bars are $\pm 1 S E M$. Large filled circles identify data points not significantly different from chance $(p>.10)$; small filled symbols identify points weakly different from chance $(.05 \leq p \leq .10)$; open circles identify points significantly different from chance $(p<.05)$. The smooth curves are the best fitting "inverted Gaussian" functions described by Equation 1, with the parameters obtained by the maximum-likelihood method (see General Method): for the broken curve, the parameter $\delta$, which characterizes the degree of loss of discriminability, was free to vary in the fitting; for the unbroken curve, $\delta$ was held fixed at .5 (chancè).

arguments against a methodological artifact will be presented later, when we present evidence about Weber fractions and data from Experiments 4 and 5, in which different psychophysical methods are shown to confirm the findings of Experiments 1 and 2.)

\section{Taste Matches}

The likelihood ratio analyses of the data of Experiments 1 and 2 are summarized in Table 1 . Overall, these analyses bear out the conclusion drawn from the simpler pointby-point chi-square tests. In 9 of the 12 individual experiments (Experiments 1.1-1.6, 2.3, 2.5, 2.6), the hypothesis that discrimination declines to chance was not rejected by the likelihood-ratio test. In each of the 3 experiments in which the likelihood-ratio statistic rejected the hypothesis with $p<.05$, there existed one or more data points near the minimum of the discrimination function that were not significantly different from chance according to the chisquare test. It is thus plausible that the magnitude of the likelihood-ratio statistic in the three cases of significant rejection is due to random deviations from the analytical form prescribed by Equation 1 rather than from failure of discrimination to decline to chance. We conclude overall that, within the concentration ranges tested, glucose, fructose, and sucrose are indiscriminable when their relative concentrations are suitably adjusted. In analogy to the well-known phenomena of color matching, we conclude that we have found taste matches for these sugars by systematic variation of their relative concentrations.

\section{A Concentration-Invariance Law for Taste Matches}

Human color matching obeys Grassmann's laws (Krantz, 1975; Wyszecki \& Stiles, 1982). One of these laws-the law of intensity invariance-states that a color match remains a match if the intensities of all components of the match are increased or decreased by the same scaling factor. The parametric analysis of the discrimination functions provided us a means of testing the hypothesis that taste matches obey a similar scaling law. In Figure 3, we plotted the MLEs of $\mu$, the "match" concentration of the glucose test stimuli against the concentrations of the fruc- 
Table 1

Maximum-Likelihood Estimates of Sugar-Discrimination Parameters Experiments 1-3: Duo-Trio Trials

\begin{tabular}{|c|c|c|c|c|c|c|c|c|c|c|c|}
\hline \multirow[b]{2}{*}{ Experiment } & \multirow[b]{2}{*}{ Subject } & \multirow[b]{2}{*}{ Standard (mM) } & \multirow[b]{2}{*}{ Test } & \multirow[b]{2}{*}{ Figure } & \multirow[b]{2}{*}{ Trials } & \multicolumn{3}{|c|}{$\omega: \delta=0.5$} & \multirow{2}{*}{$\frac{\Omega: \delta \text { free }}{\delta}$} & \multirow{2}{*}{$-2 \ln (\hat{\lambda})$} & \multirow[b]{2}{*}{$p$} \\
\hline & & & & & & $\mu(\mathrm{mM})$ & $\log _{10} \sigma^{-}$ & $\log _{10} \sigma^{+}$ & & & \\
\hline 1.1 & $\mathrm{D}$ & fructose $(100)$ & glucose & IA & 230 & 210 & 0.07 & 0.03 & .54 & 0.18 & .67 \\
\hline 1.2 & $\mathrm{C}$ & $"$ & $"$ & 1B & 210 & 210 & 0.08 & 0.07 & .44 & 1.08 & .30 \\
\hline 1.3 & $\mathrm{Y}$ & " & $"$ & $1 C$ & 320 & 200 & 0.10 & 0.07 & .54 & 0.40 & .53 \\
\hline 1.4 & $\mathrm{D}$ & fructose $(200)$ & $"$ & ID & 230 & 360 & 0.03 & 0.04 & .41 & 2.62 & .11 \\
\hline 1.5 & $\mathrm{C}$ & " & $"$ & $1 \mathrm{E}$ & 150 & 330 & 0.04 & 0.08 & .53 & 0.04 & .84 \\
\hline 1.6 & $\mathrm{Y}$ & $"$ & $"$ & $1 \mathrm{~F}$ & 130 & 400 & 0.06 & 0.04 & .64 & 1.40 & .24 \\
\hline 2.1 & $\mathrm{~T}$ & sucrose (100) & glucose & $2 \mathrm{~A}$ & 529 & 380 & 0.08 & 0.07 & .44 & 2.20 & .14 \\
\hline 2.2 & $\mathrm{R}$ & $"$ & " & $2 \mathrm{~B}$ & 500 & 340 & 0.11 & 0.09 & .40 & 6.20 & $.01^{*}$ \\
\hline 2.3 & A & $"$ & $"$ & $2 \mathrm{C}$ & 430 & 250 & 0.01 & 0.11 & .37 & 3.80 & $.05^{*}$ \\
\hline 2.4 & $\mathrm{~T}$ & sucrose $(200)$ & $"$ & $2 \mathrm{D}$ & 380 & 580 & 0.05 & 0.11 & .42 & 3.00 & .08 \\
\hline 2.5 & $\mathrm{R}$ & " & $"$ & $2 \mathrm{E}$ & 429 & 540 & 0.05 & 0.12 & .48 & 0.20 & .65 \\
\hline 2.6 & $A$ & " & " & $2 \mathrm{~F}$ & 529 & 590 & 0.08 & 0.09 & .37 & 7.60 & $.01^{*}$ \\
\hline 3.1 & $\mathrm{~B}$ & fructose (25) & maltose & $4 \mathrm{E}$ & 410 & 20 & 0.27 & 0.23 & .24 & 11.60 & $.01^{*}$ \\
\hline 3.2 & $N$ & fructose $(50)$ & " & $4 B$ & 420 & 70 & 0.02 & 0.14 & .49 & 0.01 & .92 \\
\hline 3.3 & $\mathbf{M}$ & " & $"$ & $4 C$ & 410 & 90 & 0.08 & 0.02 & .40 & 6.40 & $.01^{*}$ \\
\hline 3.4 & $\mathrm{~J}$ & fructose $(100)$ & $"$ & $4 \mathrm{~A}$ & 540 & 180 & 0.22 & 0.10 & .52 & 0.60 & .44 \\
\hline 3.5 & $N$ & $"$ & $"$ & $4 B$ & 530 & 110 & 0.06 & 0.11 & .35 & 17.80 & $.001^{*}$ \\
\hline 3.6 & $\mathrm{~B}$ & $"$ & $"$ & $4 C^{\prime}$ & 300 & 140 & 0.04 & 0.05 & .39 & 4.60 & $.05^{*}$ \\
\hline 3.7 & $\mathrm{Y}$ & $"$ & $"$ & $4 \mathrm{D}$ & 500 & 140 & 0.04 & 0.02 & .40 & 2.25 & .13 \\
\hline 3.8 & $\mathrm{M}$ & " & $"$ & $4 E$ & 530 & - & - & - & .11 & - & $* *$ \\
\hline 3.9 & $\mathrm{~J}$ & fructose $(200)$ & $"$ & $4 A$ & 320 & 280 & 0.10 & 0.23 & .35 & 6.80 & $.01^{*}$ \\
\hline 3.10 & $\mathrm{~B}$ & " & $"$ & $4 C$ & 400 & 270 & 0.03 & 0.05 & .41 & 4.20 & $.05^{*}$ \\
\hline 3.11 & $\mathrm{Y}$ & it & $"$ & $4 \mathrm{D}$ & 300 & - & - & - & .03 & - & $* *$ \\
\hline 3.12 & $\mathrm{M}$ & it & $"$ & $4 \mathrm{E}$ & 330 & - & - & - & .14 & - & $* *$ \\
\hline
\end{tabular}

Note-Each row of the table gives a summary of the data and likelihood-ratio analysis of the experiment identified in column 1. Column 2 gives the subject identification and column 3 gives the taste standard used in the experiment. Column 4 identifies the text figure containing the data. Column 5 gives the total number of discrimination trials. Columns $6-8$ give the maximum-likelihood parameter estimates (MLEs) of the inverted Gaussian function (Equation 1) for the null hypothesis space $(\omega)$ in which $\delta \equiv .5$ (see Figure 1 and General Method). Column 9 gives the MLE for $\delta$ in the larger parameter space, $\Omega$, in which $\delta$ is free to vary between 0.0 and 1.0. (Estimates for the three other parameters in $\Omega$ have been omitted for the sake of clarity; these estimates differed little from those in the restricted parameter space, $\omega$.) Column 10 gives the value of $-2 \ln (\hat{\lambda})$, where $\hat{\lambda}$ is the likelihood ratio statistic; when the null hypothesis $\delta=.5$ is true, $-2 \ln (\hat{\lambda})$ is distributed as $\chi^{2}$ Id (see General Method). Column 11 gives the significance level of the statistic for a one-tailed test, obtained from the $\chi^{2}{ }_{1 \mathrm{df}}$ distribution; a signifies a significant rejection. For Experiments 3.8,3.11, and 3.12, the parameter estimates in $\omega$ could not be determined, and the rejection is deemed highly significant $\left(^{* *}\right)$. In Experiments 1.1-2.6, the test stimuli were glucose; in Experiments $3.1-3.12$, they were maltose.

tose and sucrose standards. The slopes of the best fitting lines are not significantly different from unity (dotted lines) [sucrose, $t(4)=1.88, p>.05 ;$ fructose, $t(4)=1.98, p>$ $.05]$. Thus, over a modest threefold concentration range of glucose, taste matches obey the simplest scaling law. An additional interesting observation embodied in Figure 3 is that, within the concentration ranges of these experiments, sucrose is roughly 1.5 times as potent as fructose in producing the indiscriminable taste sensation that all three sugars produce.

\section{Experiment 3}

Rats have been shown capable of discriminating maltose from glucose and fructose, independent of concentration (Nissenbaum \& Sclafani, 1987; Spector \& Grill, 1988). In Experiments 3, we sought to determine whether human subjects could discriminate solutions of maltose and fructose.

\section{Method}

Subjects. Five female subjects served in Experiment 3. Subject $Y$ had also participated in Experiment 1.
Stimuli. Maltose (4-O- $\alpha$-D-glucopyranosyl-D-glucose; monohydrate; Sigma), a disaccharide containing two glucose molecules, and D-fructose (Aldrich) were dissolved in Naya spring water and maintained as described above.

Procedure. The procedure was generally the same as that described in General Method. However, because individual subjects exhibited distinct patterns of behavior in preliminary experiments in which maltose and fructose were paired as discriminanda, a slightly different strategy for selection of standards was adopted. All subjects initially took part in a duo-trio experiment in which the standard was $100-\mathrm{mM}$ fructose, and maltose solutions of varying concentrations served as the test stimuli. Two subjects appeared to fail to discriminate between the two sugars (Experiments 3.4 and 3.7), 2 subjects performed the discrimination at a somewhat better than chance level (Experiments 3.5 and 3.6), and 1 subject discriminated with much better than chance performance (Experiment 3.8). The subjects who performed near chance for the initial $100-\mathrm{mM}$ fructose standard were tested next with a higher concentration fructose standard $(200 \mathrm{mM}$; Experiments 3.9 and 3.11). Subjects who performed better than chance for $100-\mathrm{mM}$ fructose were tested with a lower concentration fructose standard ( $50 \mathrm{mM}$; Experiments 3.2 and 3.3). The 1 subject who performed much better than chance in the initial experiment was tested with a considerably lower concentration fructose standard $(25 \mathrm{mM}$; Experiment 3.1). To fill in the picture of discrimination for the subject of Experiments $3.3(50 \mathrm{mM})$ and $3.8(100 \mathrm{mM})$, she was also 
Standard: $100 \mathrm{mM}$ sucrose

Standard: $200 \mathrm{mM}$ sucrose
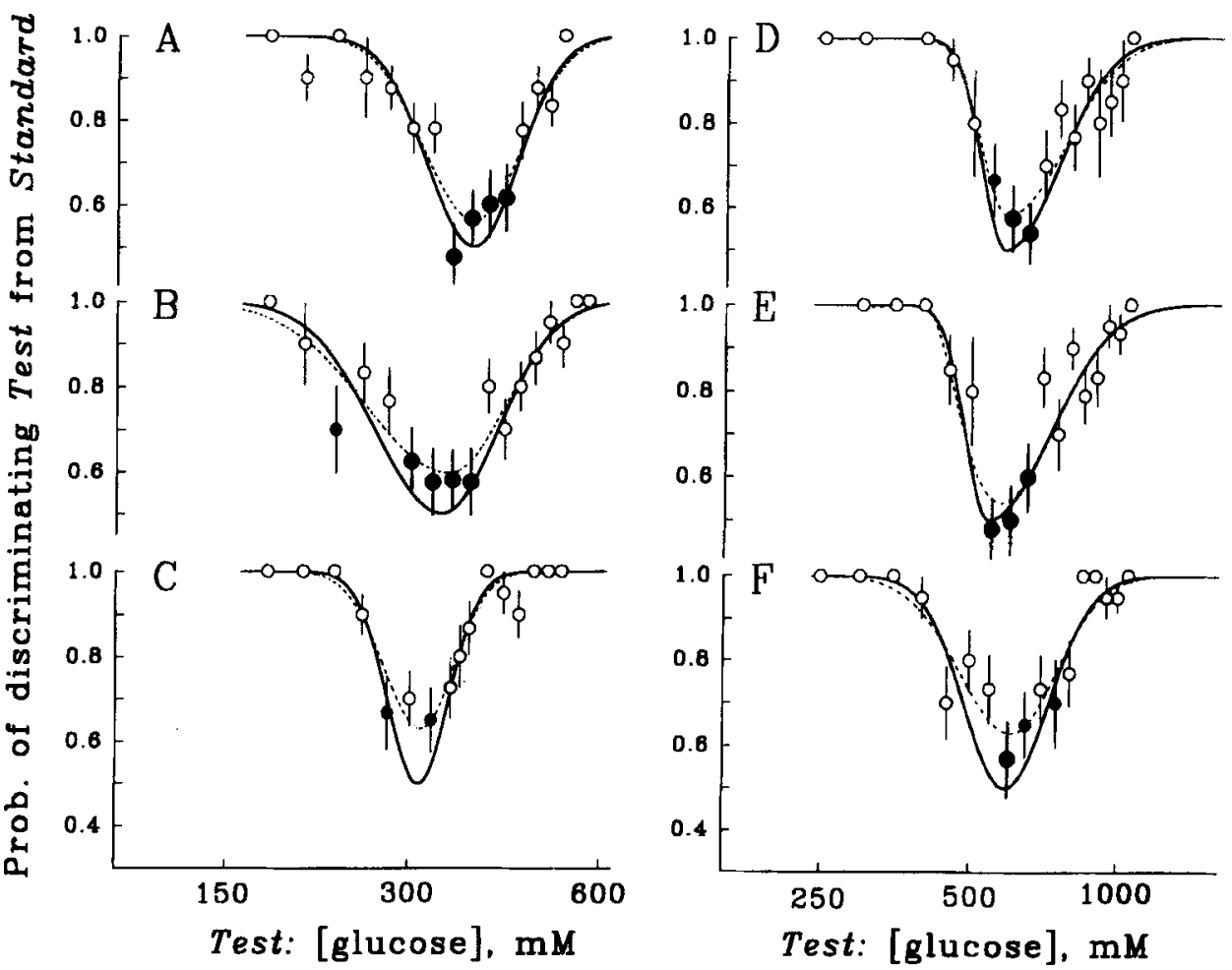

Figure 2. Discrimination functions of individual subjects for glucose test stimuli (abscissa) versus two sucrose standards (labels above panels). Each row of two panels (e.g., $A$ and $D$ ) presents the data of an individual subject. Symbols and lines have the same meanings as those in Figure 1.

tested with a $200-\mathrm{mM}$ fructose standard (Experiment 3.12). The subject who completed Experiments 3.2 and 3.5 also would have been tested with a 200 -mM fructose standard but became unavailable before that experiment could be done. The subject of Experiments 3.1 and 3.6 was tested with a 200 -mM fructose standard (Experiment 3.10) after experiments with $25-$ and $100-\mathrm{mM}$ standards were completed.

Because subjects were able to discriminate maltose and fructose at the high concentrations of the fructose standards, some of the experiments were replicated with the subjects wearing noseclips to control for olfactory cues (General Method). In these control experiments, the subjects were tested for a fixed fructose standard paired with three different maltose test stimuli: one that had previously produced the nadir of the discrimination function $(\mu)$ and two maltose test concentrations that were selected to be on each side of the nadir, at levels expected to produce perfect discrimination.

Analyses. The analyses were the same as those applied to the data of Experiments 1 and 2.

\section{Results}

Figure 4 shows results of Experiment 3. For 4 of the 5 subjects, maltose discriminability from fructose declined to chance for at least one concentration of standard, according to the chi-square and likelihood-ratio analyses (Figures $4 \mathrm{~A}-4 \mathrm{D}$, filled symbols; Table 1). In contrast to their performance with low-concentration fructose standards, 2 subjects showed nearly perfect discrimination of maltose from the 200-mM fructose standard (Figures 4D, 4E). Additional evidence for some degree of discriminability of maltose from fructose was obtained from 2 of the other 3 subjects (Figures $4 \mathrm{~A}$ and $4 \mathrm{~B}$ ). For these 2 subjects, discriminability between maltose and fructose improved with increasing concentration of the fructose standard.

For the subjects whose data are shown in Figures $4 \mathrm{C}$, $4 \mathrm{D}$, and $4 \mathrm{E}$, a subset of pairs of discriminanda were replicated with the subjects wearing noseclips. The noseclip data for the maltose test stimuli near the discrimination functions' nadirs are shown as stars ( $\star$ ). Discrimination performance was not altered by the use of noseclips.

A consistent feature across subjects of the data is that discriminability of maltose from fructose improves with the concentration of the standard. This feature of the population is illustrated in Figure 5, in which average minimum discriminability (as captured by the MLE statistic $\delta$ ) is plotted as a function of the concentration of the standard. For comparison with the maltose data, data from experiments in which glucose solutions were the test stimuli are also shown. As expected from the analysis of the results of Experiments 1 and 2, neither of the population mean $\delta$ values for glucose test stimuli is significantly different from chance. For maltose discrimination from the $100-\mathrm{mM}$ fructose standard, $\delta=0.63$, not significantly different from chance $[t(3)=2.1, p=.07]$; for maltose discrimination from 200 -mM fructose, $\delta=0.75$, significantly different from chance $[t(2)=3.01, p<.05]$. Moreover, the slope of the line through the two $\delta$ values for mal- 


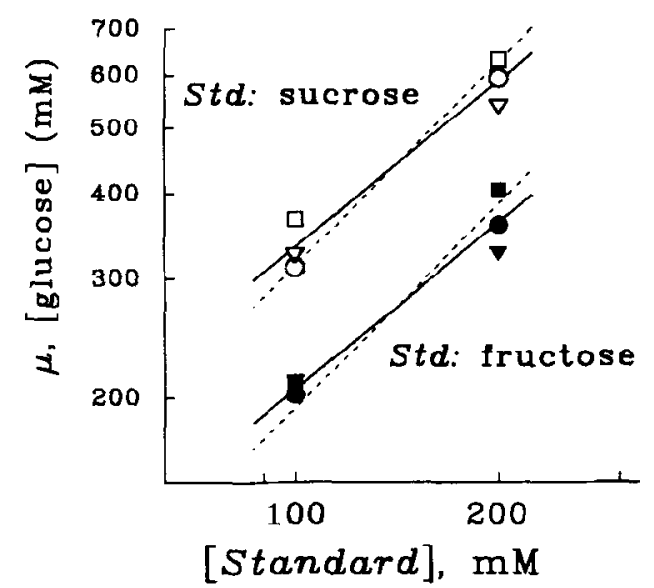

Figure 3. Estimates $(\mu)$ of the concentrations of glucose minimally discriminable from 100 - and $200-\mathrm{mM}$ fructose and sucrose standards, obtained from the maximum-likelihood analysis (Figures 1 and 2; Table 1). Open symbols identify estimates from experiments with sucrose standards, and filled symbols identify those from experiments with fructose standards; different symbols represent data from different subjects. The unbroken lines are the best fitting straight lines (least squares regression); the dashed lines are the best fitting straight lines of unity slope having the same mean value as the unbroken lines.

tose is significantly greater than zero: $m=1.2 \mathrm{M}^{-1}[t(7)=$ $3.28, p<.01]$. The latter result supports the conclusion that the discriminability of maltose from fructose improves with concentration.

In Figure 6, we plot the concentrations of maltose estimated to be minimally discriminable from the fructose standards, as a function of the standard concentration. The slope of the best fitting straight line through these maltose concentrations was not significantly different from unity. Thus, maltose "matches" obey a linear scaling relation with minimally discriminable fructose pairs over a nearly 10 -fold concentration range.

\section{Weber Fractions From Experiments 1-3}

In the light of the indiscriminability of the sugars fructose, glucose, and sucrose (and, at suitably low concentrations, maltose), the ascending and descending limbs of the discrimination functions in Figures $1-3$ can be viewed as psychometric functions for increment and decrement thresholds, and the parametric analysis of the data provides a means of estimating the Weber fractions of discrimination. Taking the $75 \%$ discrimination level as the threshold criterion, the Weber fractions obtained from Equation 1 satisfy the relationship $\pm \Delta T / T=2.71\left(\log _{10} \sigma^{ \pm}\right)$, where $T=\mu$ and $\pm \Delta T$ is the increment/decrement threshold. Averaging the 24 estimates of $\log \sigma^{+}$and $\log \sigma^{-}(\delta=.5)$ from the 12 individual experiments $1.1-2.6$, we obtain $|\Delta T| / T=0.19$ \pm 0.01 , that is, a $19 \%$ Weber fraction. This value compares well with previously reported average values for sucrose: $20 \%$ (Pfaffmann et al., 1971) and 17\% (Laing et al., 1993).

Confining attention to discrimination functions from Experiment 3 , in which the discriminability of maltose from fructose fell to chance for at least one point on the curve, and assuming transitivity of the indiscriminability relationship, we can estimate Weber fractions for maltose in terms of glucose equivalents. The average Weber fraction so obtained was $22 \%$, very close to the average values for glucose, fructose, and sucrose. Equality of the Weber fractions is expected if, at low concentrations, information about maltose is subject to the same one-dimensional coding limitation as the other three sugars.

In Figure 7, we plot the Weber fractions for all appropriate discrimination functions of Experiments 1-3. The best fitting straight line through the points has a slope insignificantly different from zero. Thus, on average, over a greater than 30-fold concentration range, Weber fractions are constant for the one-dimensional code underlying these discrimination data.

\section{EXPERIMENT 4}

A potential criticism of the duo-trio method is that the three-cup task puts a substantial memory load on the subject, and thereby obscures potentially discriminable differences between stimuli. To explore the possible limitations on discriminability imposed by the duo-trio method, we employed a simpler two-cup "same/different" procedure (Macmillan \& Creelman, 1991), and applied it to the discrimination of fructose and glucose, stimuli we had already investigated with the duo-trio task.

\section{Method}

Subjects. Three females and 1 male served in the experiment. Subject $Y$ had previously been employed in the duo-trio experiments, 1 and 3, and Subjects N, M, and J had participated in Experiment 3

Stimuli. D-Fructose and $\alpha$-D-glucose (Aldrich) were dissolved in Naya spring water and maintained as described above.

Procedure. One experimental session was run per day. In a given session, 15 pairs of small cups ( $1 \mathrm{oz}$, Baxter), each containing $10 \mathrm{ml}$ of solution, were placed before the subject. One cup of each of the 15 pairs always contained the standard solution, $200-\mathrm{mM}$ fructose. One of three stimuli formed the other member of the pair. In one subset of 5 pairs, the second stimulus was a concentration of the glucose test solution expected to be readily discriminable from the standard. In a second set of 5 pairs, the second solution was a concentration of glucose expected to be difficult to discriminate from the standard. In the third set of 5 pairs, the second stimulus was the standard itself; thus, the third set of 5 pairs were $\langle S, S\rangle$ trials. Trials from all three sets were presented in random order in each session. Moreover, the order of presentation of the members of the pairs was also randomized. In sum, each of the 15 trials in a given session consisted of successive whole-mouth tasting of 1 of 3 randomly chosen pairs of stimuli: $\left\langle S, T_{i}\right\rangle,\left\langle T_{i}, S\right\rangle,\langle S, S\rangle, i=1,2$. On each trial, the subject was required to taste the two members of the stimulus pair twice before giving a response. After tasting the pair twice, the subject was required to state whether the solutions in the two cups were the same or different and to guess when uncertain. The subject rinsed between cups, as described above.

Over days, the entire procedure was run for a series of different test stimulus concentrations, in order to complete a discrimination function. Concentrations were run in random order, with the exception that all subjects were given an initial starting concentration from the end of the concentration range and were tested repeatedly until they could discriminate the endpoint concentration from the sample 
Standard: fructose (mM): $25(0), 50(\Delta), 100(0), 200(\nabla)$
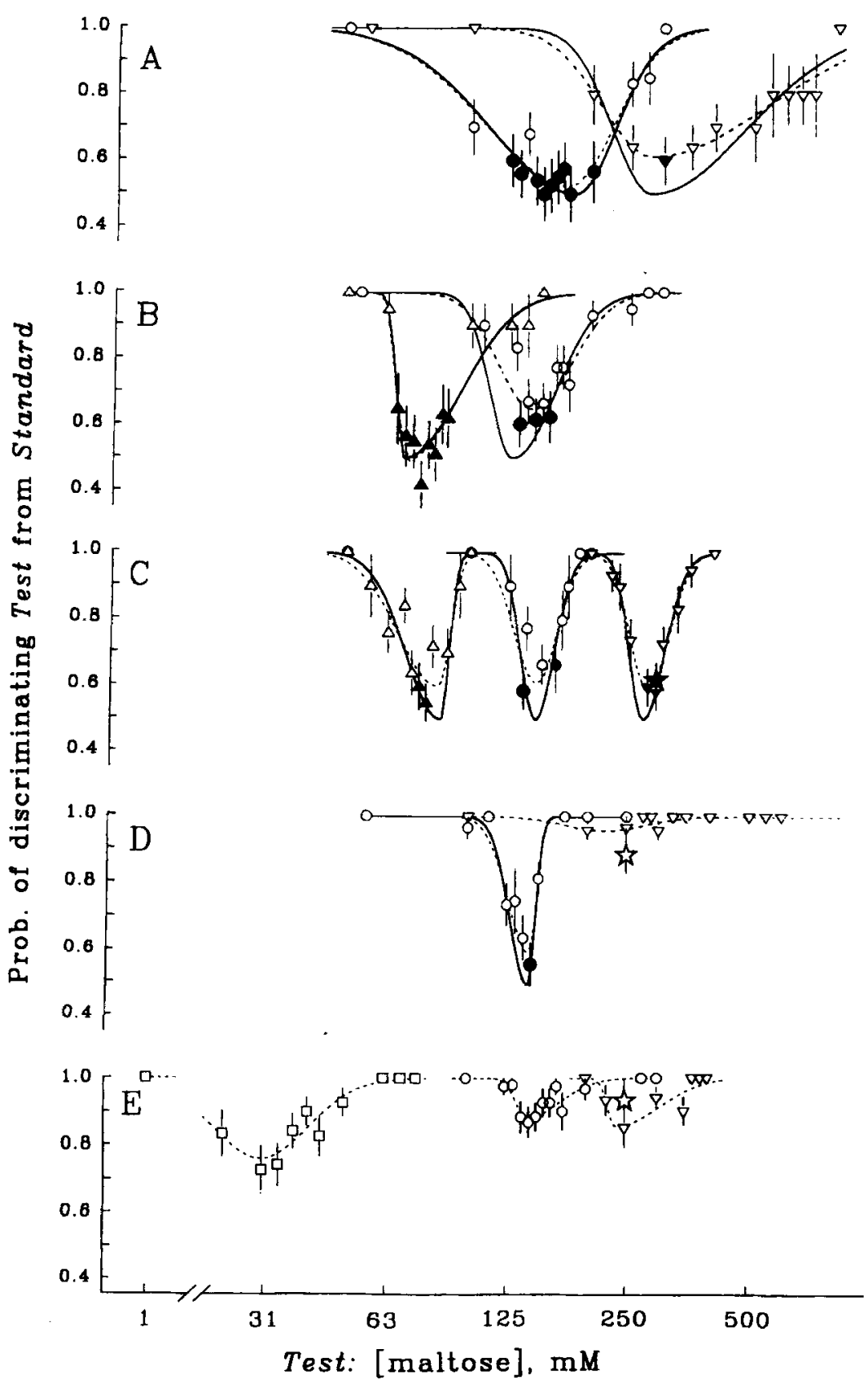

Figure 4. Discrimination functions of individual subjects for maltose test stimuli (abscissa) versus fructose standards (labels above panels) from Experiment 3. Each panel (A-E) presents the data of an individual subject with a series of different standards, as follows: $\mathrm{A}=$ subject $3.4,3.9 ; \mathrm{B}=$ subject $3.2,3.5 ; \mathrm{C}=$ subject $3.1,3.6,3.10 ; \mathrm{D}=$ subject $3.7,3.11$; and $\mathbf{E}=$ subject 3.3, 3.8, 3.12 (see Table 1). Different symbol shapes are used to denote standards of different concentrations, as follows: $\square, 25 \mathrm{mM} ; \triangle, 50 \mathrm{mM} ; \bigcirc, 100 \mathrm{mM} ; \nabla, 200 \mathrm{mM}$. The large filled symbols identify data points that do not differ significantly from chance $(p>.1)$; the smaller filled symbols identify points differing from chance weakly $(.05 \leq p \leq .10)$; open symbols represent points differing significantly from chance $(p<.05)$. The smooth curves through the points have the same significance as the curves in Figures 1 and 2. For discrimination functions in which there were no individual points (filled symbols) near chance, fitting was not done with $\delta$ held at .5 . The star symbols (open and closed) near the nadirs of the functions in Panels C, D, and E identify discrimination data obtained in sessions in which subjects wore noseclips to eliminate olfactory cues; additional data obtained with noseclips that exhibited perfect discrimination (on either side of the nadirs) are not shown. 


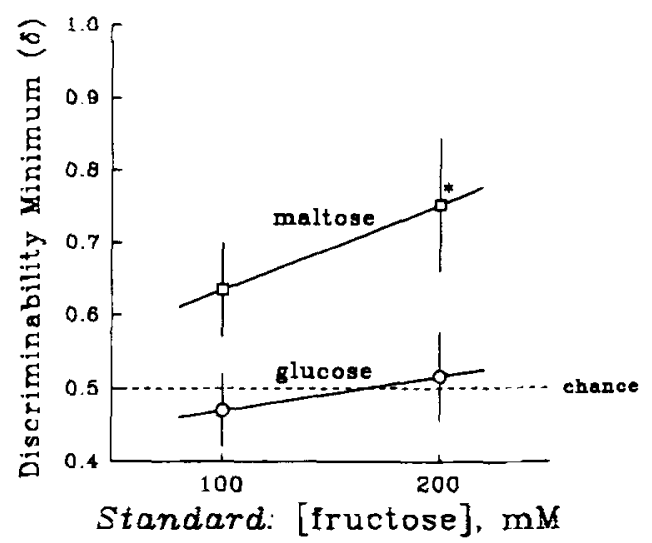

Figure 5. The MLE parameter, $\delta$, which characterizes the degree of loss of discriminability, is plotted as a function of the concentration of the fructose standards of Experiment $1(O$, glucose test stimuli) and Experiment 3 ( $\square$, maltose test stimuli). Points are means over subjects; error bars, $\pm 1 S E M$. The horizontal dashed line represents chance performance, $\delta=.5$. The unbroken lines through the points are the best fitting straight lines.

stimulus. Sets of trials that approximately resulted in chance performance were run repeatedly.

Analysis. This experiment yields discrimination functions similar to those obtained in the duo-trio task, except that response bias must be taken into consideration. One possible analysis is to treat the data for each subject as a set of $2 \times 2$ matrices in which the first-row label gives the binomial distribution of responses on the $\langle S, S\rangle$ trials and the second-row label gives the distribution of responses for trials in which the test stimulus $T_{i}$ is paired with the standard. Thus, we have $M$ response matrices of the form

$$
\begin{array}{ccc} 
& \text { "Same" } & \text { "Different" } \\
<S, S> & p \text { ("Same" } \mid<S, S>) & p \text { ("Different" } \mid<S, S>\text { ) } \\
<S, T_{i}> & \left.p \text { ("Same" } \mid<S, T_{i}>\right) & p \text { ("Different" } \mid<S, T_{i}>\text { ) }
\end{array}
$$

where the rows represent the two stimulus pairs and the columns the two possible responses. The hypothesis that discrimination declines to chance for a particular pairing $\left\langle S, T_{3}\right\rangle$ is equivalent to the hypothesis that the response distributions for the two rows are the same. We tested this hypothesis for each such matrix with the chi-square test; in plotting the data of the experiment (Figure 8), we have indicated the significance levels of these tests with different symbols.

An apparently alternative method of analysis is to transform the data for each such matrix into a $d^{\prime}$ statistic. We performed this analysis, but concluded that it provided no additional information over that gained by the simpler chi-square tests, which do not require data transformation or the ancillary "equal variance" assumption of noise-alone and signal trials (Macmillan \& Creelman, 1991)

The point-by-point chi-square analyses are less satisfactory in the same/different task because one and the same set of data constitutes the response distribution of the $\langle S, S\rangle$ trials in each of the $2 \times 2$ matrices. It follows that, unlike the case for the duo-trio data-in which the null hypothesis specifies a particular response probability, .5 , as chance-the multiple chi-square tests are not independent of one another. Nonetheless, we have presented them in Figure 7, because of their similarity to the tests used for the duo-trio data.

By a generalization of Equation 2, the results of Experiment 4 also can be analyzed with a likelihood ratio test and performance quantified with MLE parameters. The general hypothesis is now represented by a pair of equations describing the distributions of "same" and "different" responses in the two types of trials:

$$
\begin{aligned}
p \text { ("Different") } \mid<S, S>\text { ) } & =1-\delta_{0}, \\
p(\text { ("Different") } \mid<S, T>) & =1-\delta_{1} \exp \left[-\frac{1}{2}\left(\frac{\log T-\log \mu}{\log \sigma^{-}}\right)^{2}\right], T \leq \mu \\
& =1-\delta_{1} \exp \left[-\frac{1}{2}\left(\frac{\log T-\log \mu}{\log \sigma^{+}}\right)^{2}\right], T>\mu
\end{aligned}
$$

The probabilities of "same" responses in the two types of trials are the complements of the expressions given in Equation 3. The null hypothesis requires that at the test stimulus concentration $T=\mu$, the response distributions for the $\langle S, S\rangle$ and $\langle S, T\rangle$ trials be identical; the null hypothesis is captured in the parametric hypothesis $\delta_{1}=\delta_{0}$. The likelihood function for the data of Experiment 4 is similar to Equation 2, but now includes the likelihood of the response data for $<S, S>$ trials in the overall likelihood product:

$$
L\left(\mu, \sigma^{+}, \sigma^{-}, \delta_{0}, \delta_{1}, \mid \text { data }\right)=\left(1-\delta_{0}\right)^{n_{0}} \delta_{0}^{N_{0}-n_{0}} \prod_{i=1}^{M} p_{i}^{n_{i}}\left(1-p_{i}\right)^{N_{i}-n_{i}},
$$

where $N_{0}$ is the total number of $\left\langle S, S>\right.$ trials, $n_{0}$ is the number of "different" responses on $\left\langle S, S>\right.$ trials, $p_{i}=p$ ("different" $|<S, T_{i}>$ ), $N_{i}$ is the total number of $\left\langle S, T_{i}>\right.$ trials, and $n_{i}$ is the number of "different" responses on these trials. To obtain the likelihood ratio statistic, Equation 4 was maximized twice: first, over the general parameter space $\Omega$, in which $\delta_{0}$ and $\delta_{1}$ are free to vary independently of one another, and second in the parameter subspace in which $\delta_{1} \equiv$ $\delta_{0}$. As before, the siatistic $-2 \ln \hat{\lambda}$ is expected to be distributed as $\chi^{2}$ with $1 \mathrm{df}$ when the null hypothesis is true, where $\hat{\lambda}=[\max L(\omega)]$ / $[\max L(\Omega)]$.

\section{Results}

The results for 4 subjects are presented in Figure 8 . In this figure, the data points represent the values for $p_{i}=$

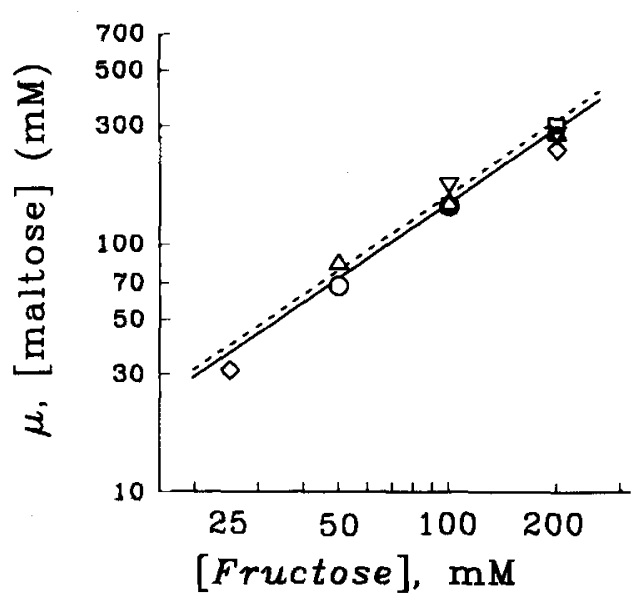

Figure 6. Estimates $(\mu)$ of the concentrations of glucose minimally discriminable from fructose standards of various concentration. The values of $\mu$ were obtained with the maximumlikelihood analysis (Figure 3; Table 1) and include estimates from discrimination functions for which minimum discrimination lay between 1.0 and chance (e.g., Figure $4 E$, all three curves). Different symbols represent results from different subjects. The unbroken line is the best fitting straight line (least squares linear regression); the dashed line is the best fitting straight line of unity slope. 


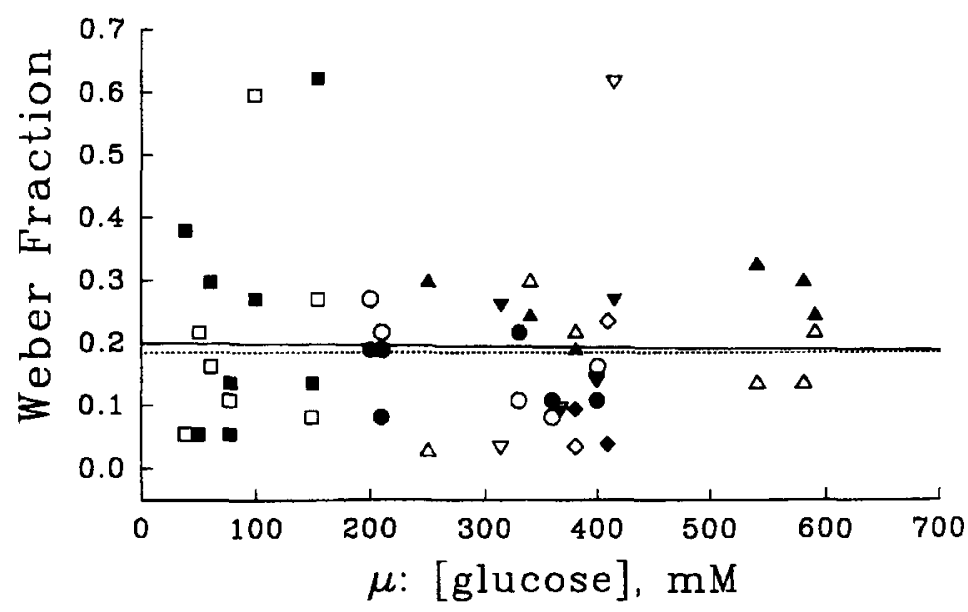

Figure 7. Weber fractions for all the discrimination functions of Experiments 1-5 plotted as a function of the "match-point concentration," $\mu$. Weber fractions were computed from $\log _{10} \sigma^{ \pm}$, the MLE steepness parameters, as described in the text. The estimates were obtained from every set of data for which performance declined to chance (Experiments 1, 2, 3, and 5), or for which the response distribution in a same/different experiment was equivalent to the response in $\langle S, S>$ trials (Experiment 4 ). The assumption of transitivity of the indiscriminability relation was used to obtain estimates of the Weber fractions from Experiment 3 with respect to a common abscissa; that is, maltose concentrations were converted into glucose equivalents for each subject. Open symbols give the Weber fractions for decrement thresholds from $\mu$, and the filled symbols give the increment thresholds. Different symbol shapes are used to denote the different experimental paradigms and standards as follows: duo-trio ( $O$, fructose; $\Delta$, sucrose; $\square$, maltose); same-different ( $\nabla$, fructose); duo-trio with feedback ( $\diamond$, fructose). The unbroken line is the straight line best fitting all the data (least squares regression). The dotted line is the best fitting line of slope zero with the same arithmetic mean as the unbroken line.

$p$ ("different" $\mid<S, T_{i}>$ ), the thicker dashed line represents the values for $p$ ("different" $\mid<S, S>$ ), and the two thinner dashed lines provide the standard error for the latter estimate. A noteworthy feature of the data is that the subjects had quite distinctive "response biases"--that is, the distribution of responses on $\langle S, S>$ trials is quite different for the different subjects (see Figure 8, insets).

Each of the discrimination functions of the 3 subjects of Figure $8 \mathrm{~A}-8 \mathrm{C}$ includes at least one point (large $\bullet$ ) which is not significantly different from the estimate of $p$ ("different" $\mid<S, S>$ ), according to the $2 \times 2$ chi-square analysis. Every data point of the discrimination function of Figure 8D rejects the hypothesis $p_{i}=p$ ("different" $\mid\left\langle S, T_{i}\right\rangle$ ) $=p$ ("different" $\mid<S, S>$ ), according to the $2 \times 2$ chi-square analysis; however, the data of Figure 8D include a point (large $O$ ) at the nadir of the function which is significantly below the thicker dashed line. While the latter data point is somewhat puzzling, it does not provide a meaningful rejection of the null hypothesis.

The curves fitted to the data in Figure 8 are those specified by Equation 3, with the parameters determined by the maximum likelihood analysis embodied in Equation 4. The MLEs for the parameters of the curves and Wilks's theorem statistics are presented in Table 2. Only the data of the subject of Figure 8B are seen to reject the hypothesis $\delta_{1}=\delta_{0}$. Since the data of Figure 8D do not reject the hypothesis (despite the point lying below the thicker dashed line) and the data of Figure $8 \mathrm{~B}$ contain one point that fails to reject the hypothesis that the response distribution is the same in $\langle S, S>$ trials, we think it reasonable to conclude that for each subject there is a region of test stimulus concentration for which the distribution of responses in $\langle S, T\rangle$ trials is the same as the distribution in $<S, S>$ trials.

There are several indications that the same/different experiments yield essentially the same information about discrimination as the duo-trio experiments. One indication is that the average value of $\mu$ obtained in the same/ different task is close to that obtained in the duo-trio task for the same standard (200-mM fructose): the means $\pm S D$ are $363 \pm 35$ and $373 \pm 44$, respectively. (Subject $Y$ was the only individual tested in both Experiment 1 and Experiment 4: her values of $\mu$ obtained for the $200-\mathrm{mM}$ fructose standard were 400 and $315 \mathrm{mM}$, respectively. Although these values are quite different, they were obtained at an interval of nearly 3 years.) A second indication comes from the Weber fractions: The average Weber fraction obtained in Experiment 4 was $22 \%$, very close to the average value (19\%) obtained in the Experiment 1 . The Weber fractions from Experiment 4 have been included in Figure 7 , and are seen to be of the same magnitude as those obtained in the duo-trio experiments. In sum, then, the somewhat simpler.same/different experiments provide evidence further consistent with the view that when the con- 


\section{Standard: $200 \mathrm{mM}$ fructose}

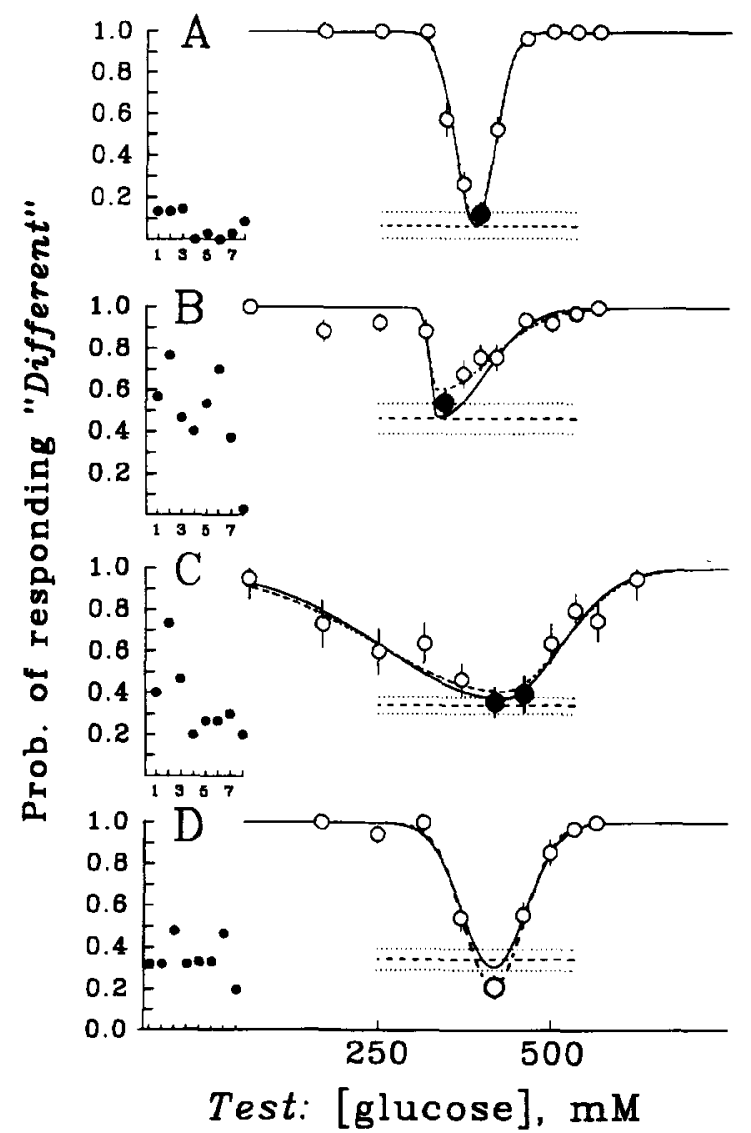

Figure 8. Same/different discrimination functions of subjects tested with a $200-\mathrm{mM}$ fructose standard and glucose test stimuli (abscissa). Each panel gives the data of a different subject (A-D). The insets at the lower left of each panel give the fractions of times the subject gave "different" responses on $\langle S, S\rangle$ trials-that is, trials in which the two stimuli were both the standard, with the data divided into eight equal bins by number of trials; these data serve to illustrate response-bias behavior over time. The dashed line is plotted at the level of the overall fraction of "different" responses on $\langle S, S\rangle$ trials; the two dotted lines about the dashed line are at $\pm 1 S E M$. Large filled symbols are data points that do not differ significantly from the dashed line $(p>.1)$; all open symbols represent points that differ from the dashed line $(p<$ $.05)$. The large open symbol in panel $D$ at the nadir of the function is significantly below the dashed line. The smooth curves are "inverted Gaussian" functions described by Equation 3, with parameters obtained by the maximum-likelihood method. The unbroken line is the curve best fitting the data, subject to the hypothesis $\delta_{0}=\delta_{1}$; the broken line is the curve best fitting the data with no constraints on the values of $\delta_{0}$ and $\delta_{1}$ (in the latter case, the MLE estimate of $\delta_{0}$ is simply the fraction of "different" responses on $\langle S, S>$ trials).

centration ratios of these sugars are appropriately adjusted, they become indiscriminable.

\section{EXPERIMENT 5}

Since subjects' detection and discrimination can improve with feedback, we tested, in this experiment, whether or not the discrimination of simple saccharides would improve with trial-by-trial feedback.

\section{Method}

Subjects. The subjects were 1 female and 2 males. Subject Mhad previously participated in Experiment 3.

Stimuli. D-Fructose and $\alpha$-D-glucose (Aldrich) were dissolved in Naya spring water and maintained as described above.

Procedure. A computer-automated duo-trio test was devised to provide trial-by-trial feedback. The trials were run as described above for Experiments 1-3, except that, after a response had been given, the computer indicated to the subject whether or not the choice was correct.

Analysis. The analysis was the same as that described above in General Method.

\section{Results}

The addition of trial-by-trial feedback improved discrimination, so that the minimum discriminability of all subjects tested was now in the $70 \%-80 \%$ range, well above chance (data not shown). Subjects in this experiment were thus obtaining and using information from the stimuli that had not been used in Experiments 1-4.

To examine the possibility that olfaction might be the source of information, all of the subjects of the preliminary feedback experiments were retested while wearing noseclips. The resultant data are shown in Figure 9. For 2 of the 3 subjects (Figures 9A and 9B), the discrimination functions now contained points not significantly different from chance according to the point-by-point chi-square analysis. The maximum likelihood analysis is reported in Table 2; it was roughly consistent with the point-by-point chi-square analysis, in that the data of Figure 9B do not reject the hypothesis, while those of Figure 9A are marginally significant $(p=.04)$. Thus, the performance of the subjects of Figures $9 \mathrm{~A}$ and $9 \mathrm{~B}$ are consistent with the hypothesis that the glucose solution, the fructose solution, or both could produce distinctive odors for subjects.

In contrast to the behavior of the subjects of Figures 9A and 9B, the subject whose data are shown in Figure 9C consistently performed above chance, even with a noseclip. We conclude that this subject can use more than one oral sensory signal in performing the task. In the General Discussion, we consider the nature of information that he might have used. We note, however, that even this subject exhibited a very clear minimum in discriminability at a stimulus concentration very near that of the minima of the other 2 subjects.

An indication of the improvement of discriminability of subjects when tested in the trial-by-trial feedback paradigm is that the Weber fractions declined. For the subjects of Figures $9 \mathrm{~A}$ and $9 \mathrm{~B}$, the average Weber fraction was 0.082 , less than $1 / 2$ the Weber fractions obtained in the other experiments (see Figure 7).

\section{GENERAL DISCUSSION}

\section{Monogeusia for Simple Saccharides}

The experiments and analyses presented here show that solutions of glucose, fructose, and sucrose - and, at low 
Table 2

Maximum-Likelihood Estimates of Sugar-Discrimination Parameters

A. Experiment 4: Same/Different Trials

\begin{tabular}{|c|c|c|c|c|c|c|c|c|c|c|c|c|}
\hline \multirow[b]{2}{*}{ Experiment } & \multirow[b]{2}{*}{ Subject } & \multirow[b]{2}{*}{ Standard (mM) } & \multirow[b]{2}{*}{ Figure } & \multirow{2}{*}{$\begin{array}{c}\text { Trials } \\
\text { Diff/Same }\end{array}$} & \multirow[b]{2}{*}{$\mu(\mathrm{mM})$} & \multicolumn{2}{|c|}{$\omega: \delta_{0} \equiv \delta_{1}$} & \multirow[b]{2}{*}{$\delta_{0}=\delta_{1}$} & \multicolumn{2}{|c|}{$\Omega: \delta_{0} \& \delta_{1}$ free } & \multirow[b]{2}{*}{$-2 \ln (\hat{\lambda})$} & \multirow[b]{2}{*}{$p$} \\
\hline & & & & & & $\log _{10} \sigma^{-}$ & $\log _{10} \sigma^{+}$ & & $\delta_{0}$ & $\overline{\delta_{1}}$ & & \\
\hline 4.1 & $\mathrm{~N}$ & fructose $(200)$ & $8 \mathrm{~A}$ & $530 / 270$ & 366 & 0.03 & 0.03 & 0.93 & 0.93 & 0.89 & 0.60 & $\overline{\text { n.s. }}$ \\
\hline 4.2 & $\mathrm{Y}$ & " & $8 B$ & $500 / 250$ & 315 & 0.01 & 0.08 & 0.53 & 0.54 & 0.40 & 7.50 & $.01 *$ \\
\hline 4.3 & $\mathrm{M}$ & " & $8 \mathrm{C}$ & $260 / 130$ & 414 & 0.21 & 0.10 & 0.62 & 0.65 & 0.58 & 1.20 & n.s. \\
\hline 4.4 & $\mathrm{~J}$ & $"$ & $8 D$ & $395 / 220$ & 399 & 0.05 & 0.05 & 0.69 & 0.66 & 0.78 & 5.20 & $.01 *$ \\
\hline
\end{tabular}

B: Experiment 5: Duo-Trio Trials With Trial Feedback

\begin{tabular}{|c|c|c|c|c|c|c|c|c|c|c|}
\hline \multirow[b]{2}{*}{ Experiment } & \multirow[b]{2}{*}{ Subject } & \multirow[b]{2}{*}{ Standard $(\mathrm{mM})$} & \multirow[b]{2}{*}{ Figure } & \multirow[b]{2}{*}{ Trials } & \multicolumn{3}{|c|}{$\omega: \delta \equiv 0.5$} & \multirow{2}{*}{$\frac{\Omega: \delta \text { free }}{\delta}$} & \multirow[b]{2}{*}{$-2 \ln (\hat{\lambda})$} & \multirow[b]{2}{*}{$p$} \\
\hline & & & & & $\mu(\mathrm{mM})$ & $\log _{10} \sigma^{-}$ & $\log _{10} \sigma^{+}$ & & & \\
\hline 5.1 & $\mathrm{R}$ & fructose $(200)$ & $9 \mathrm{~A}$ & 330 & 411 & 0.08 & 0.001 & 0.37 & 7.60 & $.01^{*}$ \\
\hline 5.2 & M & $"$ & $9 \mathrm{~B}$ & 300 & 381 & 0.01 & 0.03 & 0.46 & 0.60 & .78 \\
\hline 5.3 & $\mathrm{G}$ & " & $9 \mathrm{C}$ & 350 & 392 & 0.01 & 0.03 & 0.31 & 10.40 & $.01^{*}$ \\
\hline
\end{tabular}

Note-Each row of the table summarizes the data and analysis of the individual experiment identified in column 1. Part A summarizes Experiment 4; Part B summarizes Experiment 5. For part B, the columns have the same identification as those of Table 1. For the analysis of Experiment 4 , the larger parameter space, $\Omega$, includes separate $\delta$ parameters for $\langle S, T\rangle$ and $\langle S, S\rangle$ trials (columns 10 and 11 ), as described in the text; the remaining columns have the same interpretation as that in Table 1.

concentrations, maltose-are indiscriminable to most human subjects when their relative concentrations are suitably adjusted. In analogy with the monochromacy of night vision, whereby any two lights are indiscriminable when their relative intensities are suitably adjusted, we call the gustatory indiscriminability of these simple sugars monogeusia. The finding of monogeusia implies that, for these sugars in the concentration ranges studied, there is a one-dimensional "bottleneck" in the neural path between the chemical receptors and the gustatory percept. One reasonable hypothesis about the identity of the bottleneck is that it occurs at the level of the gustatory chemical receptors themselves, just as the monochromacy of scotopic vision is explained by the hypothesis that rods containing rhodopsin are the only photoreceptors producing detectable signals in the fully dark-adapted retina (see Pugh, 1988). If, in fact, the bottleneck occurs at the receptor level, so that these sugars bind reversibly to a single class of membrane receptor in taste cells, then our data yield estimates of the relative affinities of the binding site for the four sugars. Thus, on the assumptions that the binding is weak and first order, and that the psychophysical indiscriminability relation is transitive, we find the relative affinities for low-to-moderate concentrations of sucrose, maltose, fructose, and glucose "at the mucosal surface" to be $1,3 / 4,2 / 3,1 / 3$, respectively. It is also possible that information is represented by many neural signals that are kept in a constant ratio as their (joint) magnitude is varied-for instance, multiple channels equated simultaneously.

Monogeusia for the simple sugars resembles scotopic monochromacy in that monogeusia obeys a concentrationinvariance, or "scaling," law (Figures 3,6). Indeed, were it not for the obedience to the scaling law, the relative affinities estimated in the previous paragraph would not be concentration invariant.

\section{Breakdown of Monogeusia with Maltose}

The breakdown of monogeusia in the experiments in which maltose was tested against higher concentration fructose standards is important both experimentally and theoretically. Experimentally, the breakdown provides additional evidence that the failures of discrimination that define monogeusia are not artifactual, that is, not due to limitations imposed by the methodology or the power of the statistical tests.

Theoretically, the breakdown of monogeusia with maltose is important because it suggests that maltose at high concentrations activates at least one additional neural code over that activated by the other sugars. This conclusion rests on the hypothesis that monogeusia at low concentrations occurs because only one gustatory channel is activated by all four sugars. Thus, a parsimonious explanation of all the maltose data is this: at low concentrations, maltose interacts effectively only with the same receptor site with which glucose, fructose, and sucrose interact; at higher concentrations, however, maltose interacts with a second class of receptor that gives rise to a second, and perceptually distinct, neural signal. However, this was more apparent in some subjects' maltose data (Figures 4D and 4E) than in others'. Among individuals, there was great variability in the degree to which monogeusia broke down for maltose.

The ability of humans to discriminate maltose from the other sugars is consistent with the findings of recent discrimination experiments on rats (Nissenbaum \& Sclafani, 1987; Spector \& Grill, 1988). Our experiments extend the work on rats by demonstrating that concentrationindependent discrimination does not require olfactory cues (Figure 4, stars). Indeed, some subjects spontaneously reported that at higher concentrations maltose took on a "malty" taste and also had a longer aftertaste that permitted them to make the discrimination. (None were aware that maltose was a stimulus.) 


\section{Standard: $200 \mathrm{mM}$ fructose}

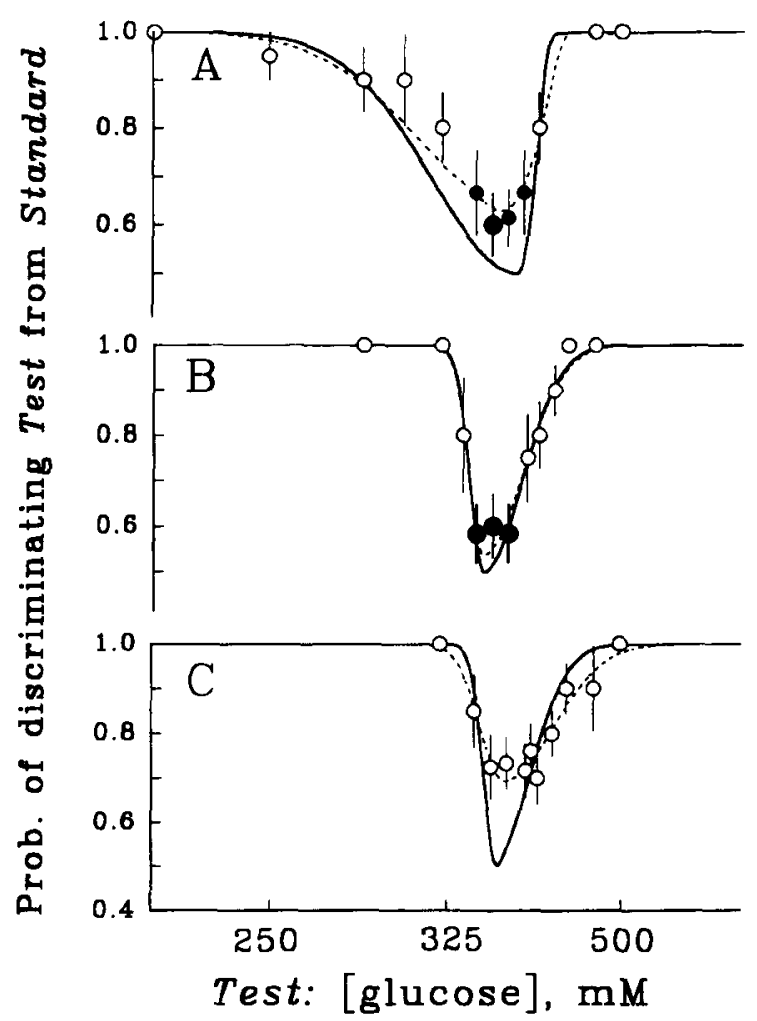

Figure 9. Discrimination functions of individual subjects wearing noseclips while performing the duo-trio task for a $200-\mathrm{mM}$ fructose standard and glucose test stimuli (abscissa). Each panel (A-C) presents the data of an individual subject. Symbols and lines have the same significance as those in Figures 1-2. The abscissa in this figure has been expanded relative to that in Figures $1,2,4$, and 8 .

A particularly interesting feature of the maltosediscrimination data is the obedience to the scaling law of the maltose concentrations minimally discriminable from fructose, even at levels where monogeusia has failed (Figure 6). An interpretation of this scaling behavior is that even at stimulus concentrations of maltose sufficiently high to activate the secondary neural code or codes, the primary neural channel continues to provide a dominant concentrationdependent signal. Discriminability declines to its minimum when the primary signal produced by the maltose test and the fructose standard are equated, leaving the subject with only the signal(s) from the secondary code(s) to make a discrimination (e.g., Figure 4E). Classical color-matching experiments, which are usually not performed as discrimination experiments, typically do not yield information about the mechanisms underlying discriminability of nonmatching stimuli. However, recent color-vision experiments in which forced-choice discrimination has been employed have yielded results quite similar to those presented here, in that minima in discrimination functions were found that likely reflect the equating of the signals of a primary neural code while signals from a secondary code were used for discrimination (Calkins et al., 1992).

\section{Methodology in Taste-Discrimination Experiments}

The results reported here have several implications for the choice of psychophysical methods to be used in tastediscrimination experiments. Perhaps the most important conclusion to be drawn is that the fundamental question of the number and nature of the sensory codes in taste is unlikely to be resolved without the use of forced-choice psychophysical techniques. Had we only used "simple matching" procedures in which subjects adjusted the relative concentrations of the sugars until they reported that they "matched" (as is normally done in color matching), neither we nor anyone else would have accepted the notion that the sugars investigated produced truly indiscriminable neural signals.

While the use of forced-choice methodology is time consuming, it nonetheless yields rich payoffs beyond defining equivalence classes of indiscriminable stimuli. For example, the formal analysis of the discrimination functions can yield Weber fractions (Figure 7) and even information about the behavior of the neural codes when more than one code is functioning in the discrimination behavior (Figures 4 and 6).

The relatively simpler same/different task can yield much the same information as the more complex and timeconsuming duo-trio experiments, as demonstrated in the population match points $(\mu \mathrm{s})$ and Weber fractions resulting from the data of Experiment 4 . However, the data of the same/different experiments also underscore the wellknown and powerful effects of response bias. One feature of such experiments that can make statistical interpretation difficult is that response bias may not be stationary over a series of trials collected over a period of many days (insets, Figure 8).

Experiment 5, in which feedback was provided in the duo-trio task, sounds a note of caution about some of the conclusions based upon Experiments 1-4. Certainly, the results of Experiment 5 call for additional investigations with feedback, and emphasize the need to be concerned about olfactory cues. Moreover, while the possibility of a gustatory cue subserving discrimination in Experiment 5 cannot be excluded, there are at least two obvious nongustatory cues that could subserve the stably improved discriminability of the subject of Figure $9 \mathrm{C}$ - solution osmolarity and solution viscosity. Though neither of these cues is likely to be encoded by gustatory neurons, it seems plausible that both cues could give rise to differential neural activity in neurons in the mouth. Viscosity, for example, could affect vibration-sensitive neurons by means of altering the slip friction of the tongue in the buccal cavity. Indeed, since fructose, glucose, and sucrose exhibit different viscosity at the same sweetness levels, testing at higher concentrations than used in the present experiments would be impractical to use due to the high-viscosity differences. Osmoreceptors are widely present throughout the body, and could be 
present in the mouth. Experiments are now being designed to attempt to isolate these cues in order to determine their significance.

\section{Von Skramlik's Geschmacksgleichungen (Taste Matching)}

Von Skramlik (1921a, 1921b, 1926) conducted an extensive series of taste-matching experiments with salts, employing methods similar to those used by Helmholtz (1896) to examine color matching. The salts he used were reported to elicit a rich variety of different taste sensations: for example, $\mathrm{MgCl}_{2}$ elicited a bitter-salty taste, $\mathrm{K}_{2} \mathrm{SO}_{4}$, a bittersour taste, $\mathrm{NaHCO}_{3}$, a bitter-sweet taste, and $\mathrm{BeSO}_{4}$, a sour-sweet taste. Subjects in von Skramlik's experiments were asked to compare a salt solution with mixtures of four stimuli-quinine- $\mathrm{HCl}, \mathrm{NaCl}$, tartaric acid, and glucoseand to indicate whether the salt solution and the mixture tasted the same. Von Skramlik reported that for every salt stimulus he tested it was possible to find a suitable mixture of the four stimuli such that subjects said the mixture tasted the same as the test salt solution. On the basis of von Skramlik's findings, we would say, in the terms we have adopted, that human subjects are tetrageusic for his salt stimuli. Since forced-choice procedures were not used, however, von Skramlik's work cannot be regarded as conclusive by today's psychophysical standards (for a discussion of forced-choice efficacy, see Rafal, Smith, Krantz, Cohen, \& Brennen, 1990). Nonetheless, his work suggests that the total number of neural signals encoding gustatory stimuli is small.

In contrast to the simplicity of von Skramlik's view, others have concluded that there are probably many distinctive taste receptors (Bartoshuk, 1987). However, much of the psychophysical evidence that has been taken to support the notion of a multiplicity of distinct codes for sweet-tasting compounds has been obtained from taste thresholds and cross-adaptation experiments. The sensorycomparative viewpoint may be illuminating here. In human color vision, Stiles's $(1939,1953,1978)$ classic two-colorincrement threshold studies yielded incontrovertible evidence for at least five, and possibly seven distinct " $\pi$ mechanisms" subserving photopic thresholds and color discrimination. Nonetheless, for his discovery of the multiplicity of $\pi$ mechanisms, Stiles (1967) never doubted the secure evidence from metameric color matching that only three classes of cone photoreceptors initially encode all photopic stimuli. The $\pi$ mechanisms are now known to be consequences of the variety of postreceptor pathways that signals - originating in only three classes of cones - follow to the higher visual centers (reviewed in Pugh \& Kirk, 1986).

The challenge for the future will be to determine if von Skramlik was, in fact, correct that the number of neural signals encoding sapid stimuli is small, and to determine, with modern psychophysical methods, whether or not even complex-tasting compounds-such as $\mathrm{KCl}$, which is described as bitter and salty, or saccharin, which is described by some as bitter and sweet - can be rendered indiscriminable from suitable mixtures of a small number of other taste stimuli.

\section{REFERENCES}

Bartoshuk, L. M. (1987). Is sweetness unitary? An evaluation of the evidence for multiple sweets. In J. Dobbing (Ed.), Sweetness (pp. 33-43). New York: Springer-Verlag.

Beidler, L. M., \& TonosaKi, K. (1985). Multiple sweet receptor sites and taste theory. In D. W. Pfaff (Ed.), Taste, olfaction, and the central nervous system (pp. 47-64). New York: Rockefeller University Press.

BirCH, G. G. (1987). Chemicals aspects of sweetness. In J. Dobbing (Ed.), Sweetness (pp. 3-14). New York: Springer-Verlag.

Breslin, P. A. S., KemP, S., \& Beauchamp, G. K. (1994). Single sweetness signal. Nature, 369, 447-448.

Calkins, D. J., Thornton, J. E., \& Pugh, E. N., Jr. (1992). Monochromatism determined at a long-wavelength/middle-wavelength cone-antagonistic locus. Vision Research, 32, 2349-2367.

ERICKSON, R. P. (1982). Studies on the perception of taste: Do primaries exist? Physiology \& Behavior, 28, 57-62.

Faurion, A. (1987). Physiology of the sweet taste. Progress in Sensory Physiology, 8, 129-201.

Helmholtz, H. von (1896). Handbuch der physiologischen Optik [Handbook of physiological optics] (2nd ed.). Hamburg: Voss.

Hettinger, T. P., Myers, W. E., \& Frank, M. E. (1990). Role of olfaction in perception in non-traditional "taste" stimuli. Chemical Senses, 15, 755-760.

Jakinovich, W., \& Sugarman, D. (1989). Peripheral mechanisms of mammalian sweet taste. In R. J. Cagan (Ed.), Neural mechanisms in taste (pp. 37-83). Boca Raton, FL: CRC Press.

Kemp, S. E., \& Beauchamp, G. K. (1994). Flavor modification by sodium chloride and monosodium glutamate. Journal of Food Science, $59,682-686$.

Krantz, D. H. (1975). Color measurement and color theory: I. Representation theory for Grassman structures. Journal of Mathematical Psychology, 12, 283-303.

laing, D. G., Prescott, J., Bell, G. A., Gillmore, R., James, C., Best, D. J., Allen, S., Yoshida, M., \& Yamazaki, K. (1993). A cross-cultural study of taste discrimination with Australians and Japanese. Chemical Senses, 18, 161-168.

Lawless, H. T., \& Stevens, D. A. (1983). Cross adaptation of sucrose and intensive sweeteners. Chemical Senses, 7, 309-315.

Macmillan, N. A., \& Creelman, C. D. (1991). Detection theory: A user's guide. New York: Cambridge University Press.

MCBurNEY, D. H. (1972). Gustatory cross adaptation between sweettasting compounds. Perception \& Psychophysics, 11, 225-227.

Nissenbaum, J. W., \& Sclafani, A. (1987). Qualitative differences in polysaccharide and sugar tastes in rats: A two-carbohydrate taste model. Neuroscience \& Biobehavioral Reviews, 11, 187-196.

Pfaffmann, C., Bartoshuk, L. M., \& McBurney, D. H. (1971). Taste psychophysics. In L. M. Beidler (Ed.), Handbook of sensory physiology: Vol. 4. Chemical senses: Part 2. Taste (pp. 75-101). New York: Springer-Verlag.

Pugh, E. N., JR. (1988). Vision: Physics and retinal physiology. In R. C Atkinson, R. J. Herrnstein, G. Lindzey, \& R. D. Luce (Eds.), Stevens Handbook of experimental psychology: Vol. I. Perception and motivation (pp. 75-164). New York: Wiley.

Pugh, E. N., JR., \& KIRK, D. B. (1986). The $\Pi$ mechanisms of W. S Stiles: An historical review. Perception, 15, 705-728.

Rafal, R., Smith, J., Krantz, J., Cohen, A., \& Brennen, C. (1990). Extrageniculate vision in hemianopic humans: Saccade inhibition by signals in the blind field. Science, 250, 118-121.

Schiffman, S. S., Cahn, H., \& Lindley, M. G. (1981). Multiple receptor sites mediate sweetness: Evidence from cross adaptation. Pharmacology, Biochemistry \& Behavior, 15, 377-388.

Shallenberger, R. S. (1993). Taste chemistry. London: Blackie

SPECTOR, A. C., \& GRILL, H. J. (1988). Differences in the taste quality of maltose and sucrose in rats: Issues involving the generalization of conditioned taste aversions. Chemical Senses, 13, 95-113.

Stiles, W. S. (1939). The directional sensitivity of the retina and the spectral sensitivity of the rods and cones. Proceedings of the Royal Society of London: Series $B, 127,64-105$.

STILES, W. S. (1953). Further studies of visual mechanisms by the twocolour threshold technique. In Colloquio sobre problemas opticos de 
la vision (Vol. 1, pp. 65-103). Madrid: Union Internationale de Physique Pure et Appliquée.

STILES, W. S. (1967). Mechanism concepts in colour theory. Journal of the Colour Group, 11, 106-123.

STILES, W. S. (1978). Mechanisms of colour vision. London: Academic Press.

von SKramlik, E. (1921a). Mischungsgleichungen im Gebiete des Geschmacksinns [Mixture equations for the sense of taste]. Zeitschrift für Psychologie und Physiologie der Sinnesorgane, 53 (2 Abt.), 36-78. von Skramlik, E. (1921b). Mischungsgleichungen im Gebiete des Geschmacksinns [Mixture equations for the sense of taste]: II. Zeit- schrift für Psychologie und Physiologie der Sinnesorgane, 53 (2 Abt.), 219-233.

von Skramlik, E. (1926). Handbuch der Physiologie der niederen Sinne die Physiologie des Geruchs- und Geschmackssinnes [Handbook of physiology for the "lower" senses: The physiology of smell and taste] Leipzig: G. Thieme.

WILKS, S. S. (1962). Mathematical statistics. London: Wiley.

WYSZECKI, G., \& STILES, W. S. (1982). Color science. New York: Wiley.

(Manuscript received March 6, 1995;

revision accepted for publication August 7, 1995.) 